\title{
Performance Improvement of 4x4 Extended Alamouti Scheme with Implementation of Eigen Beamforming Technique
}

\author{
Maharshi N. Rindani \\ Lecturer, EC Department \\ RK University, Rajkot, |ndia-360007
}

\author{
Nishchal M. Rindani \\ Sr. Lecturer, EC Department \\ A.V.P.T.I., Rajkot, India- 360001
}

\begin{abstract}
This paper highlights on the error performance improvement of $4 \times 4$ Extended Alamouti Scheme with implementation of Eigen-Beamforming technique. In a MIMO communication system, the beamforming technique improves the Signal-toNoise Ratio (SNR) and/or Signal-to-Interference-plus-Noise ratio. So, to get advantage of beamforming technique, $4 \times 4$ Extended Alamouti Scheme is implemented with Eigen beamforming technique. This combined technique comprises of channel state information which is fed back to the transmitter side in order to achieve proper error performance as compared to Extended Alamouti Scheme without Beamforming. This proposed scheme provides array gain by increasing the bit-error-rate (BER) performance for different wireless communication channels and M-PSK modulation scheme.
\end{abstract}

\section{Keywords}

MIMO, BER, SVD, EAC, PSK

\section{INTRODUCTION}

If we want to build any digital communication system with high data rates, then we need to increase the order of the modulation scheme, because the data rate of the QPSK modulation scheme is higher than that of the BPSK modulation scheme. At the same time as we move towards the higher order modulation scheme, along with increased in data rates, error rates are also increased. So, if we want to achieve small error rates than we need to implement MIMO transmission systems [9].

Mainly three types of antenna configurations available those are SIMO (Single Input Multiple Output), MISO (Multiple Input Single Output) and MIMO (Multiple Input Multiple Output).

When there is only one transmitting antenna and more than one receiving antennas are available in any communication system than that system is known as Single Input Multiple Output (SIMO) system, shown in figure-1(a). If a communication system consisting of more than one transmitting antennas and one receiving antennas than that system is known as Multiple Input Single Output (MISO) communication system, shown in figure-1(b). Similarly if a communication system consisting of more than one transmitting as well as receiving antennas than that system is known as MIMO (Multiple Input Multiple Output), shown in figure-1(c). [11], [12], [16]

Now, if we want to use these multiple antenna configurations than there should be proper transmission schemes. To decrease the error rate and to increase the data rate and signal to noise ratio are available three transmission schemes: Spatial Multiplexing, Diversity and Beamforming, see figure-2 [5].

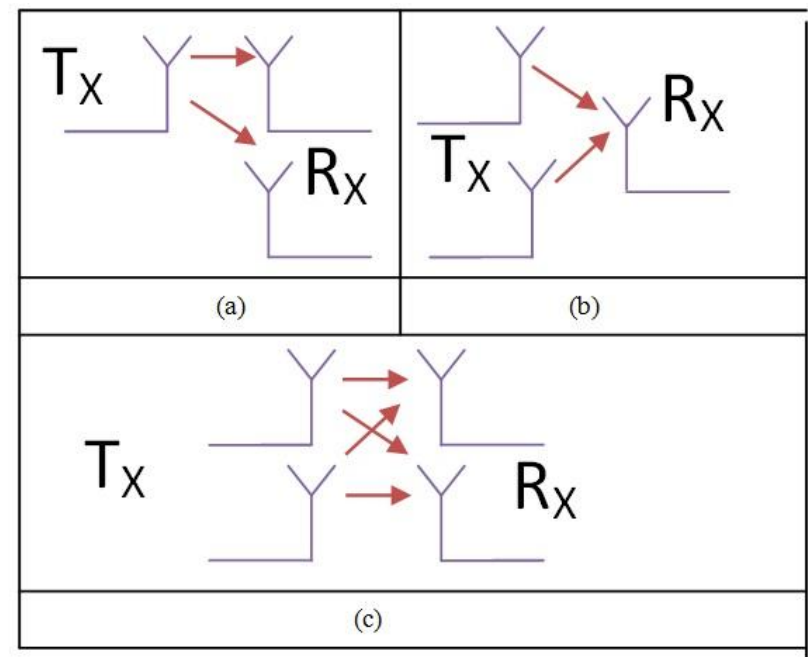

Fig 1: Different Antenna Configurations

By using SPATIAL MULTIPLEXING MIMO transmission scheme, we get benefit in improvement in data rate. That is achieved by improvement in MULTIPLEXING GAIN. By use of BEAMFORMING, we get improvement in SNR and that achieve by increment in ANTENNA GAIN. DIVERSITY technique used to decrease the error rate by increasing CODING GAIN as well as DIVERSTIY GAIN.

There is a tradeoff between all these gains. So, if we want to overcome this tradeoff, then the combined technologies are useful [1]. 


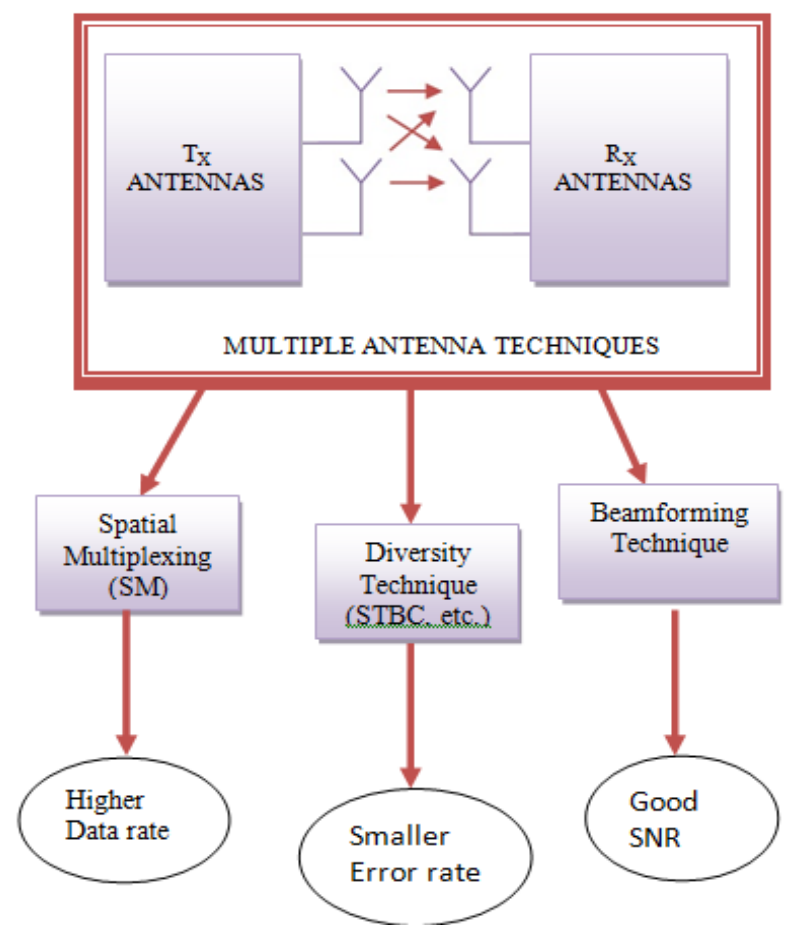

Fig 2: MIMO Transmission Scheme

\section{EXTENDED ALAMOUTI CODING}

As discussed in [7], we can improve the error performance by transmitting same symbols for more than one time by using the concepts of coding and interleaving. In [7], one of the well known space time transmitting for $2 \times 1$ diversity order as well as hybrid for $2 \times 2$ diversity order are described. Now, in [15], basic idea of extension in Alamouti scheme provided, which has obtained by extending it for 4x1-diversity order. Now, here it further extended for 4x4-diversity order [17].Now, consider block diagram representation of EAC (Extended Alamouti Code) shown in figure-3.

"Firstly, four symbols S1, S2, S3 \& S4 are given to the STBC encoder, which uses the concept of coding and interleaving. The symbols are transmitted by different antennas through different paths of the same wireless channel.. Similarly during second time-slot its coded symbols such as $\mathrm{S} 2 *,-\mathrm{S} 1 *, \mathrm{~S} 4 *$ and $-\mathrm{S} 3 *$ are being transmitted and so on.

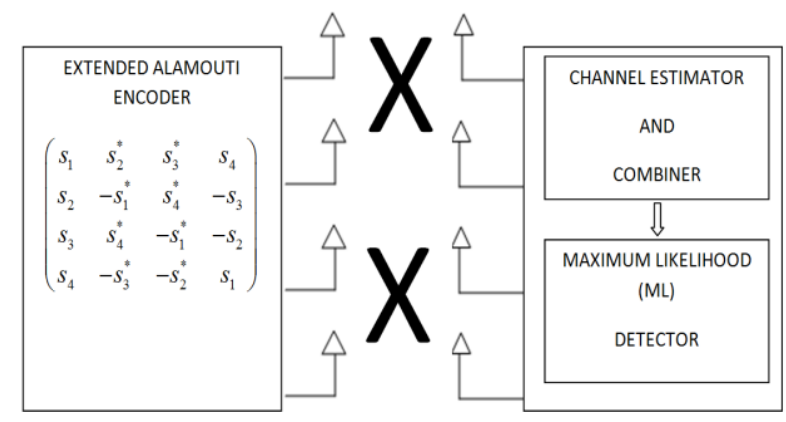

\section{Fig 3: Extended Alamouti Scheme}

All transmitted coded symbols passed through the wireless communication channel and this channel is generally represented by channel matrix shown in equation-(1):-
$\mathrm{H}=\left[\begin{array}{llll}\mathrm{h}_{1,1} & \mathrm{~h}_{1,2} & \mathrm{~h}_{1,3} & \mathrm{~h}_{1,4} \\ \mathrm{~h}_{2,1} & \mathrm{~h}_{2,2} & \mathrm{~h}_{2,3} & \mathrm{~h}_{2,4} \\ \mathrm{~h}_{3,1} & \mathrm{~h}_{3,2} & \mathrm{~h}_{3,3} & \mathrm{~h}_{3,4} \\ \mathrm{~h}_{4,1} & \mathrm{~h}_{4,2} & \mathrm{~h}_{4,3} & \mathrm{~h}_{4,4}\end{array}\right]$

where hi,j represents the fading coefficient of the propagation path from the ith transmit antenna to the $\mathrm{jth}$ receive antenna, with $i=1 . . N T, j=1 . . N R$.

These all transmitted symbols are being received at EAC decoder / EAC decoder, which are given by the following well-known equation:-

\section{$\mathrm{R}=\mathrm{H}^{*} \mathrm{~S}+\eta$}

Where $\mathrm{S}$ represents the transmitting symbol matrix as shown in figure, $\mathrm{H}$ represents the channel matrix as shown in equation-(1) and $\eta$ is AWGN (Additive White Gaussian Noise) [16]. All these received symbols are given to the channel estimator and combiner section of decoder portion of MIMO communication system. These section estimates the channel parameter from the received symbols and given to the combiner section. Now outputs of combiner and channel estimator portion are given by -

$\tilde{\mathrm{S}}=\mathrm{H}^{\dagger} * \mathrm{Y}$

Where $\mathrm{Y}$ and $\mathrm{H}+$ are represented by equation(4) and (5), respectively. Now, this $\hat{s}$ represents the symbols detected at the receiver side, which are being compared with the transmitted symbols $\mathrm{S}$.

$\mathbf{H}^{\dagger}=\left(\begin{array}{cccc}\mathrm{h}_{1,1} & \mathrm{~h}_{1,2} & \mathrm{~h}_{1,3} & \mathrm{~h}_{1,4} \\ \mathrm{~h}_{2,1} & \mathrm{~h}_{2,2} & \mathrm{~h}_{2,3} & \mathrm{~h}_{2,4} \\ \mathrm{~h}_{3,1} & \mathrm{~h}_{3,2} & \mathrm{~h}_{3,3} & \mathrm{~h}_{3,4} \\ \mathrm{~h}_{4,1} & \mathrm{~h}_{4,2} & \mathrm{~h}_{4,3} & \mathrm{~h}_{4,4} \\ -\mathrm{h}_{2,1}^{*} & \mathrm{~h}_{1,1}^{*} & -\mathrm{h}_{1,4}^{*} & \mathrm{~h}_{1,3}^{*} \\ -\mathrm{h}_{2,2}^{*} & \mathrm{~h}_{2,1}^{*} & -\mathrm{h}_{2,4}^{*} & \mathrm{~h}_{2,3}^{*} \\ -\mathrm{h}_{3,2}^{*} & \mathrm{~h}_{3,1}^{*} & -\mathrm{h}_{3,4}^{*} & \mathrm{~h}_{3,3}^{*} \\ -\mathrm{h}_{4,2}^{*} & \mathrm{~h}_{4,1}^{*} & -\mathrm{h}_{4,4}^{*} & \mathrm{~h}_{4,3}^{*} \\ -\mathrm{h}_{1,3}^{*} & -\mathrm{h}_{1,4}^{*} & \mathrm{~h}_{1,1}^{*} & \mathrm{~h}_{1,2}^{*} \\ -\mathrm{h}_{2,3}^{*} & -\mathrm{h}_{2,4}^{*} & \mathrm{~h}_{2,1}^{*} & \mathrm{~h}_{2,2}^{*} \\ -\mathrm{h}_{3,3}^{*} & -\mathrm{h}_{3,4}^{*} & \mathrm{~h}_{3,1}^{*} & \mathrm{~h}_{3,2}^{*} \\ -\mathrm{h}_{4,3}^{*} & -\mathrm{h}_{4,4}^{*} & \mathrm{~h}_{4,1}^{*} & \mathrm{~h}_{4,2}^{*} \\ \mathrm{~h}_{1,4}^{*} & \mathrm{~h}_{1,3} & \mathrm{~h}_{1,2} & \mathrm{~h}_{1,1} \\ \mathrm{~h}_{2,4} & \mathrm{~h}_{2,3} & \mathrm{~h}_{2,2} & \mathrm{~h}_{2,1} \\ \mathrm{~h}_{3,4} & \mathrm{~h}_{3,3} & \mathrm{~h}_{3,2} & \mathrm{~h}_{3,1} \\ \mathrm{~h}_{4,4} & \mathrm{~h}_{4,3} & \mathrm{~h}_{4,2} & \mathrm{~h}_{4,1}\end{array}\right)$ 
$\mathrm{Y}=\left(\begin{array}{l}\mathrm{R}^{1} \\ \mathrm{R}^{2^{*}} \\ \mathrm{R}^{3^{*}} \\ \mathrm{R}^{4}\end{array}\right)$

\section{EXTENDED ALAMOUTI CODING WITH BEAMFORMING TECHNIQUE}

Beamforming is one of the well-known MIMO transmission techniques implemented in order to improve the error performance by improving Signal-to-Interference-Noise Ratio (SINR) or Signal-to-Noise Ratio (SNR).

In case of the MIMO beamforming technique, we are providing information regarding angles at which they received at the antennas. It is necessary to provide these information, because signals are coming at the receiver through multipath fading effect which causes the signals to come at different time and angles. In addition, there is necessary to provide beamforming-weighting factors in order to provide proper shape to RF antennas. [9], [2].

Now, this angle information is developed by taking angle of the exponential of the channel fading parameter [18]. Similarly, weighting factor can be obtained by Eigen beamforming method.

Now, this Eigen beamforming method is based on SVD (Singular Value Decomposition) method. If we generate SVD of channel matrix $\mathrm{H}$ (shown in equation- (1)), than it results into following equation-(6);

$\operatorname{SVD}\{[\mathrm{H}]\}=\mathrm{U} * \mathrm{D} * \mathrm{U}^{\mathrm{T}}$

Where, D represents the diagonal matrix that represents the system as equivalent parallel SISO channel.U is the nonorthogonal matrices, which play important roles in the formation of the beamforming weights. [4]

Now, implementation and combination of MIMO Eigen Beamforming technique with EAC is shown in figure - (4). Its Mathematical description is also shown below-

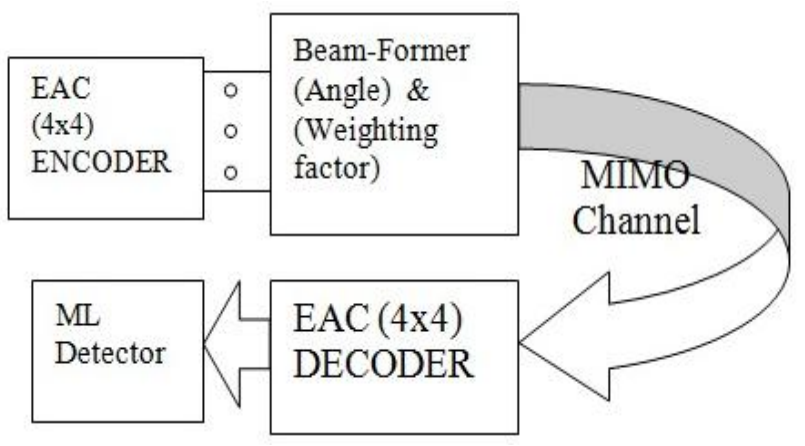

Fig 4: EAC with Beamforming Technique
Now, received signal at EAC Decoder is given by -

$$
\mathrm{y}=\mathrm{H}^{*} \mathrm{~W} * \Theta * \mathrm{~S}+\eta
$$

Where, $\mathrm{y}$ is the received signal, $\mathrm{H}$ is the channel matrix as shown in equation-(1). $\mathrm{S}$ is the transmitted symbol matrix given by equation- (8). $\eta$ is AWGN added during the transmission of the symbols through fading channel.

$$
S=\left(\begin{array}{cccc}
s_{1} & s_{2}^{*} & s_{3}^{*} & s_{4} \\
s_{2} & -s_{1}^{*} & s_{4}^{*} & -s_{3} \\
s_{3} & s_{4}^{*} & -s_{1}^{*} & -s_{2} \\
s_{4} & -s_{3}^{*} & -s_{2}^{*} & s_{1}
\end{array}\right)
$$

Here, $\mathrm{W}$ is the beamforming weighting matrix, which is equal to the matrix $U$ which represents Eigen vectors. This provides the channel state information back to the transmitter side. Now, $\Theta$ is the angle information that is the inverse of the phase of the channel that is multiplied with weighting factor to ensure that the signals add constructively at the receivers [18]. That angle information in mathematical form is shown in below equation $-(9)$ :

$$
\Theta=\left(\begin{array}{llll}
e^{-i \theta_{11}} & e^{-i \theta_{21}} & e^{-i \theta_{31}} & e^{-i \theta_{41}} \\
e^{-i \theta_{12}} & e^{-i \theta_{22}} & e^{-i \theta_{32}} & e^{-i \theta_{42}} \\
e^{-i \theta_{13}} & e^{-i \theta_{23}} & e^{-i \theta_{33}} & e^{-i \theta_{43}} \\
e^{-i \theta_{14}} & e^{-i \theta_{24}} & e^{-i \theta_{34}} & e^{-i \theta_{44}}
\end{array}\right)
$$

Now, these received signals are given to the decoder and at last to the ML detector. To measure the error performances for various channels such as AWGN channel and RAYLEIGH and NAKAGAMI fading channels, the detected symbols are compared with the transmitted symbols.

\section{SIMULATION RESULTS \& DISCUSSION}

This section comprises of the simulation results of the ALAMOUTI 2x2 scheme as shown in [1] with beamforming, EAC with and without beamforming technique for M-PSK modulation schemes for various wireless fading channels.

Here, figure-(5), (6) shows the results for Rayleigh fading channels and M-PSK modulation schemes. As shown in these figures, Extended Alamouti Code for $4 \mathrm{x} 4$-diversity order with beamforming provides better results as compared to the Alamouti $2 \times 2$ with beamforming technique.

Along with that, it can be seen from the figures EAC with beamforming provides better coding as well as diversity gain as compared to the EAC without beamforming technique. Therefore, we can say that as the fading effect is higher, this combining technique provides better error performance because of CSI (Channel State Information) feed back to the transmitter side. 


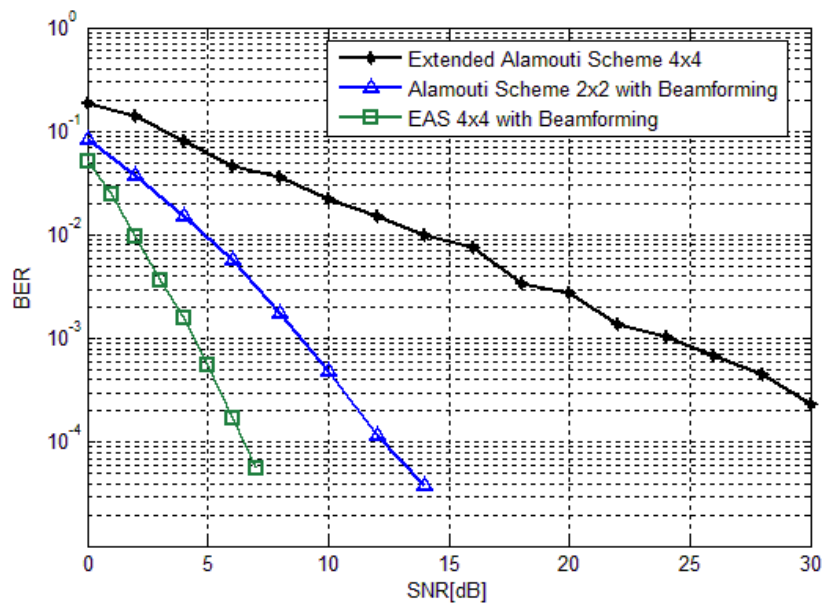

Fig 5: BER analysis for Rayleigh fading channel, BPSK Modulation Scheme

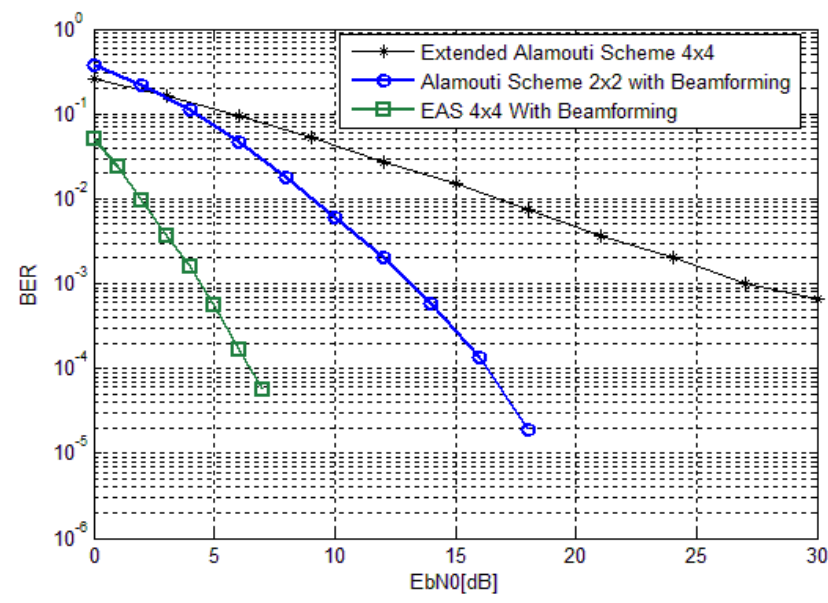

FIg 6: BER analysis for Rayleigh fading channel, QPSK Modulation Scheme

Similarly, as the modulation scheme order is increased from ' 2 ' to ' 4 ', data rate is increased. Therefore, along with it error rate is also increased. Therefore, error performance is degraded.

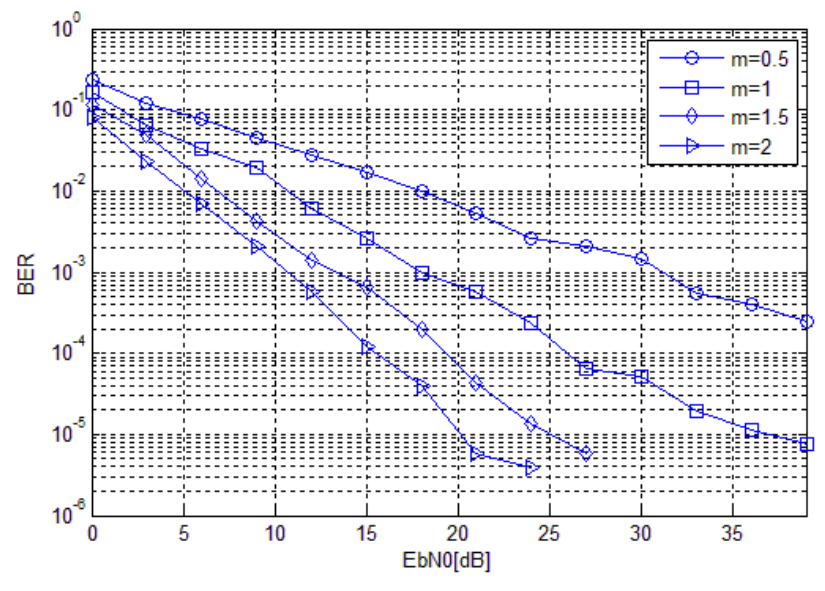

Fig 7: BER analysis for NAKAGAMI fading channel, BPSK Modulation Scheme (EAC)

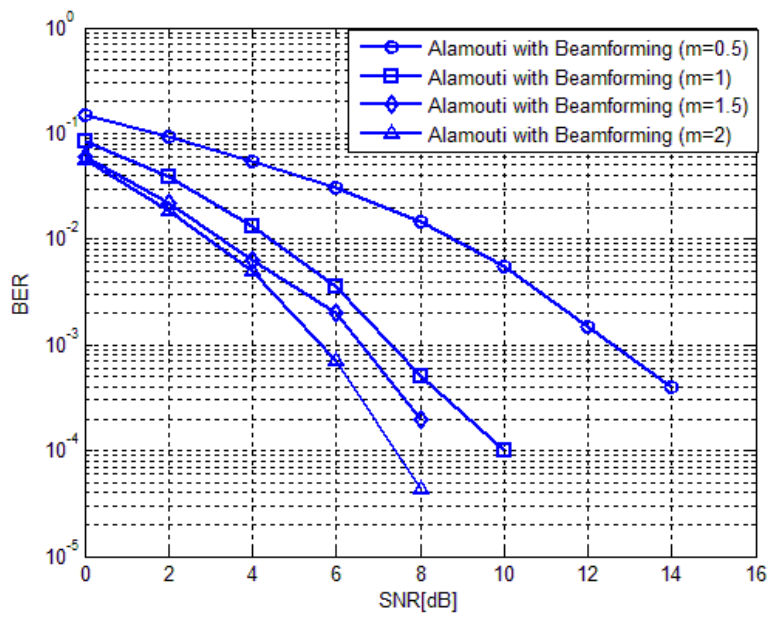

Fig 8: BER analysis for NAKAGAMI fading channel, BPSK Modulation Scheme (Alamouti $2 \times 2$ with BF)

Now, figures 7 to 12 shows the error performances for the NAKAGAMI fading channel for M-PSK modulation scheme for Extended Alamouti Scheme, EAC 4x4 with Beamforming and Alamouti scheme with beamforming, respectively. Now, as the value of nakagami factor is increasing from $m=0.5$ to $\mathrm{m}=2$, its error performance is improved for each and every modulation schemes and diversity techniques. Now, if we compare EAC with and without Beamforming techniques for M-PSK modulation schemes than EAC with beamforming provides better results as compared to the EAC without beamforming as shown in Table-1.

Table-1. BER ANALYSIS FOR QPSK

\begin{tabular}{|c|c|c|c|}
\hline & \multirow{2}{*}{$\begin{array}{c}\text { Nakagami } \\
\text { Fading } \\
\text { Channel }\end{array}$} & \multicolumn{2}{|c|}{ BER Values } \\
\hline $\mathbf{S}$ & & $\begin{array}{c}\text { EAC } \\
4 \times 4\end{array}$ & $\begin{array}{c}\text { EAC 4X4 + } \\
\text { BF }\end{array}$ \\
\hline \multirow{2}{*}{ At $\mathrm{SNR}=5 \mathrm{~dB}$} & $\mathrm{~m}=0.5$ & 0.22885 & 0.1302 \\
\hline & $m=2$ & 0.059231 & 0.0186 \\
\hline \multirow{2}{*}{ At $S N R=10 d B$} & $\mathrm{~m}=0.5$ & 0.1359 & 0.0563 \\
\hline & $\mathbf{m}=2$ & $\mathbf{0 . 0 1 0 5 7 7}$ & 0.0077 \\
\hline
\end{tabular}

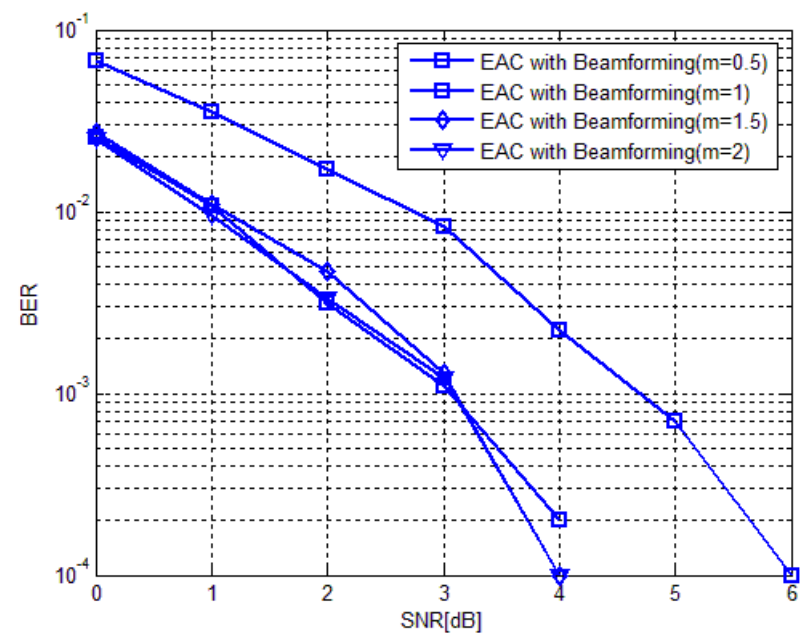

Fig 9: BER analysis for NAKAGAMI fading channel, BPSK Modulation Scheme (EAC with BF) 


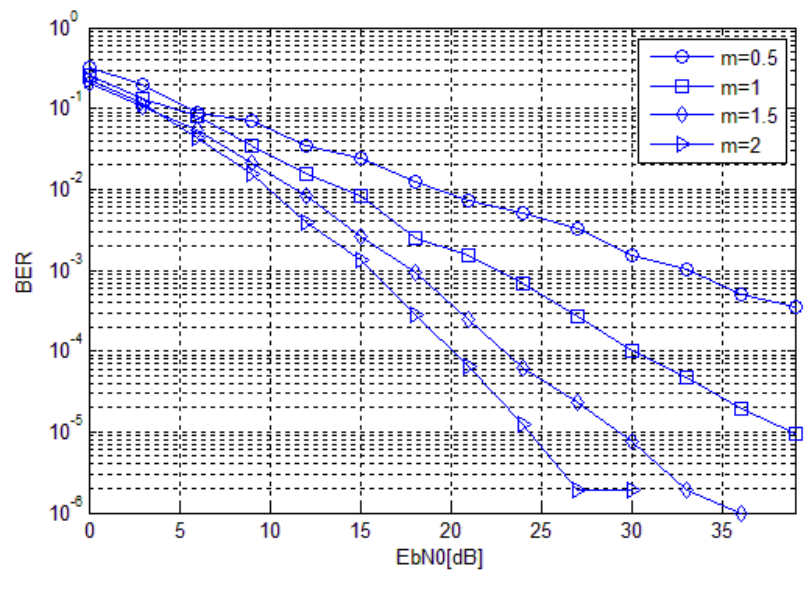

Fig 10: BER analysis for NAKAGAMI fading channel, QPSK Modulation Scheme (EAC)

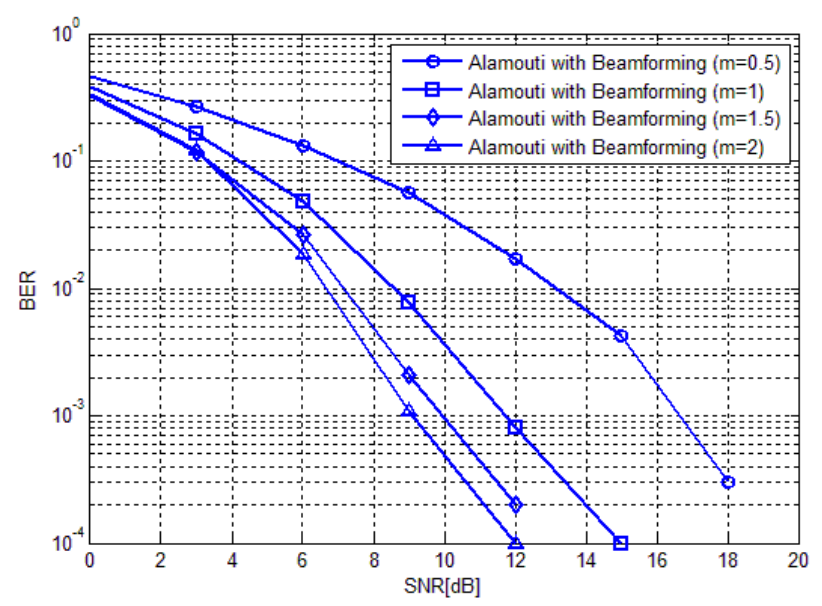

Fig 11: BER analysis for NAKAGAMI fading channel, QPSK Modulation Scheme (Alamouti 2x2 with BF)

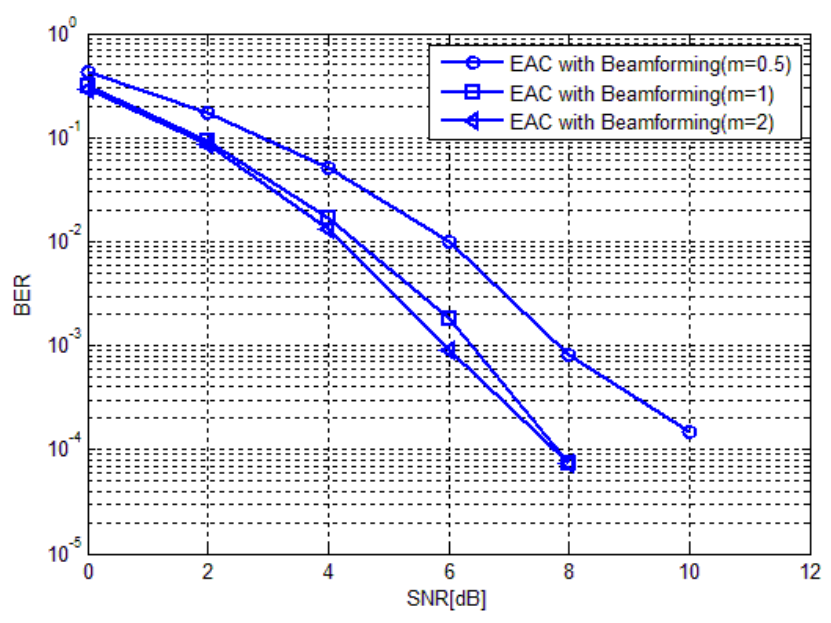

Fig 12: BER analysis for NAKAGAMI fading channel, QPSK Modulation Scheme (EAC with BF)

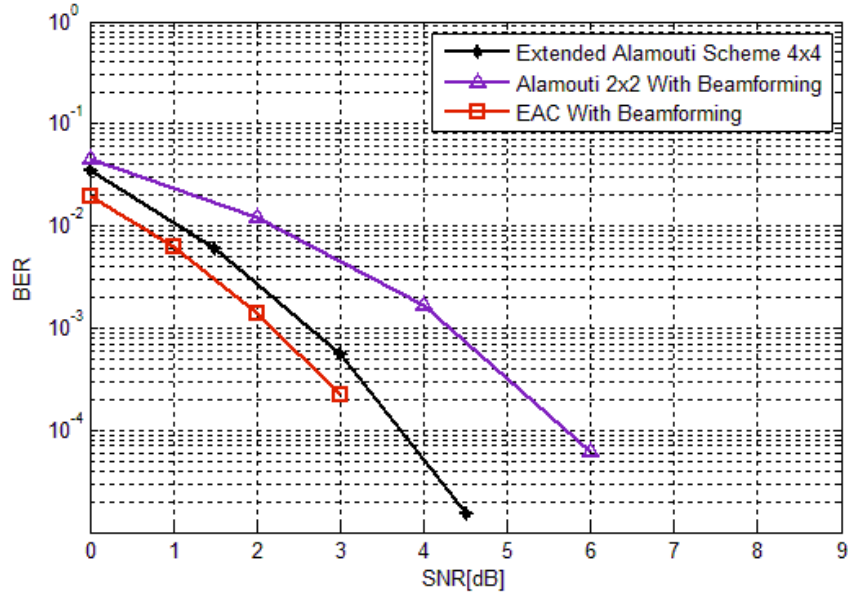

Fig 13: BER analysis for AWGN channel, BPSK Modulation Scheme

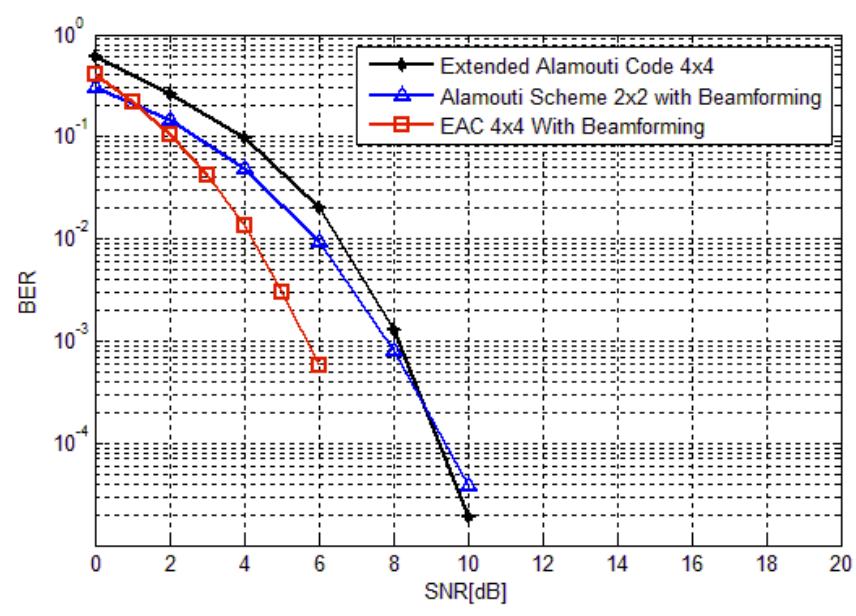

Fig 14: BER analysis for AWGN channel, QPSK Modulation Scheme

Now, figure-13 and 14 provide the results for the all proposed schemes for AWGN channel and M-PSK modulation schemes. Here also proposed scheme i.e. EAC with Beamforming provides the better error performance as compared to other diversity schemes.

\section{CONCLUSION}

This Paper provides new proposed scheme, which is combination of Extended Alamouti Code and EigenBeamforming technique for various wireless communication channels such as AWGN, RAYLEIGH \& NAKAGAMI fading channels. As shown in section of result and analysis, this new proposed combined scheme of EAC $4 \times 4$ with beamforming provides the better error performance as compared to others. Here, error performance is improved not only in terms of coding gain but also in terms of diversity gain. 


\section{REFERENCES}

[1] Jan Mietzner, " Multiple-Antenna Systems", (Accessed: 12 October 2012).

[2] D. Tse and P. Viswanath, Fundamentals of Wireless Communication, Cambridge University Press, USA, 2005

[3] Y. S. Cho, J. Kim, W. Y. Yang, C. G. Kang,"MIMOOFDM wireless communications with MATLAB", John Wiley \& Sons, Ltd., 2010.

[4] J. G. Proakis and M. Salehi, Communications Systems Engineering. Upper Saddle River, NJ: Prentice-Hall, 1994, pp. 265-267.

[5] S. M. Alamouti, "A simple transmit diversity scheme for wireless communications," IEEE Journal on Selected Areas in Communications pp. 1451-1458, October 1998.

[6] C. F. Mecklenbrauker, M.Rupp, "Generalized Alamouti Codes for Trading Quality of Service against Data Rate in MIMO UMTS", EURASIP Journal on Applied Signal Processing 2004:5, 662-675.

[7] M. Rindani, A. Bavarva, "BER PERFORMANCE COMPARISON OF 4X4 EXTENDED ALAMOUTI SCHEME FOR DIFFERENT FADING CHANNELS", International Journal of Advanced Research in Electrical, Electronics and Instrumentation Engineering (IJAREEIE) Vol. 2, Issue 3, March 2013.

[8] Jin Liu, Erry Gunawan, "Exact Bit-error rate analysis for the combined system of Beamfoming and Alamouti's Space-Time block code", IEEE Microwave and wireless components Letters, Vol. 14, No.08, August 2004.

[9] Krishna Shankar, "Transmit Beamforming" [Online],Available:http://www.dsplog.com/2009/04/13/tr ansmit-beamforming/ (Accessed date : - 21March, 2013).

[10] John Fitzpatrick, "Simulation of a Multiple Input Multiple Output (MIMO) wireless system", Dublin City University School of Electronic Engineering, April 2004.
[11] Shengli zhou, Georgies B. Giannakis, "Optimal Transmitter Eigen Beamforming and Space Time Block Coding Based on Channel Mean Feedback", IEEE Transaction on Signal Processing, Vol. No. 50, No.10, October 2002.

[12] George Jongren, Mikael skaglund, Bjorn attersten, "Combining beamforming and Space Time Block coding ", IEEE Transactions on information theory, Vol. 48, No. 3, March 2002.

[13] Daniel Figueiredo, Muhammad Imadur Rahaman, Nicola Marchetti, Frank H.P. Fitzek, Maras D. Katz, Youngkwan Cho, Ramjee Prasad, "Transmit Diversity Vs. Beamforming for Multi-User OFDM Systems", 4G Research Lab, Samsung Electronics Co. Ltd. , Korea, 2004.

[14] Peerapoang Uthansakal, Apinya Innok and Monthippa Uthansakal, "Open-loop Beamforming Technique for MIMO system and its Pracitcal Realization", International journal of Antenna and Propogation Volume, 2011.

[15] F. H. Gregorio , "SPACE TIME CODING FOR MIMO SYSTEMS", Helsinki University of Technology, Signal Processing Laboratory,POB 3000, FIN-02015 HUT, Finland

[16] H. Jafarkhani, "A Quasi Orthogonal Space-Time Block Code,” IEEE Trans. Comm., vol. 49, Jan. 2001, pp. 1-4

[17] C. Langton, B. Sklar, "Tutorial 27 - Finding MIMO", [online] Oct 2011, http://www.complextoreal.com (Accessed: 12 August 2012).

[18] L. Kansal, A. Kansal and K. Singh, "Performance Analysis of MIMO-OFDM System Using QOSTBC Code Structure for M-QAM", Canadian Journal on Signal Processing Vol. 2, No. 2, May 2011. 